\title{
A SPECTRAL CONDITION DETERMINING THE KAEHLER PROPERTY
}

\section{HAROLD DONNELLY}

ABSTRACT. We prove that the spectrum of the reduced complex Laplacian determines if a Hermitian manifold is Kaehler.

In [1] Marcel Berger proposed the problem of determining from the spectrum of the real Laplacian whether or not a manifold is Kaehler. It would seem natural to consider, rather than the real Laplacian, some differential operator containing information about the Hermitian structure. Peter Gilkey [3] has recently proved the Berger conjecture for the complex Laplacian.

Our paper considers the reduced complex Laplacian of [6], and proves the Berger conjecture for this operator.

We would like to thank V. K. Patodi for acquainting us with the problem, and Peter Gilkey for informing us of his invariance theory results which are used essentially. Finally we wish to acknowledge the encouragement given to us by Professor Chern.

The Einstein convention of summing over repeated indices will be employed throughout. Further we denote by $A$ the multi-index $a_{1} \cdots a_{p}$, and by $B$ the multi-index $b_{1} \cdots b_{q}$.

1. The reduced complex Laplacian. Let $M^{d}$ be a compact Hermitian manifold with Hermitian metric $h$. Define $g(X, Y)$ to be the real part of $b(X, Y)$. Then $g(X, Y)$ is a Riemannian metric on the tangent bundle of $M$. We extend $g$ to be conjugate linear on the complexified tangent bundle, and set $g_{\alpha \beta}=g\left(\partial / \partial z_{\alpha}, \partial / \partial z_{\beta}\right)$ with inverse matrix $g^{\alpha \beta}$ in any local coordinate system.

Define $\nabla$ to be the canonical Hermitian connection on the complexified tangent bundle. Then we have the following formulas for covariant differentiation [4]:

$$
\nabla_{\partial / \partial z_{\alpha}}\left(\frac{\partial}{\partial z_{\beta}}\right)=\sum \Gamma_{\alpha \beta}^{\gamma} \frac{\partial}{\partial z_{\gamma}}, \quad \nabla_{\dot{\partial} / \partial \bar{z}_{\alpha}}\left(\frac{\partial}{\partial z_{\beta}}\right)=0, \quad 1 \leq \alpha, \beta \leq d,
$$

Received by the editors October 30, 1973 and, in revised form, December 15, 1973.

AMS (MOS) subject classifications (1970). Primary 53C99.

Copyright $\odot$ 1975, American Mathematical Society 
where

$$
\Gamma_{\alpha \beta}^{\gamma}=\sum \frac{\partial g_{\beta \epsilon}}{\partial z_{\alpha}} g^{\boldsymbol{\gamma}} .
$$

If $K$ is the curvature tensor, then define

$$
\begin{aligned}
& K\left(\frac{\partial}{\partial z_{\alpha}}, \frac{\partial}{\partial \bar{z}_{\beta}}\right)\left(\frac{\partial}{\partial z_{\gamma}}\right)=\sum K_{\gamma \alpha \bar{\beta}}^{\epsilon} \frac{\partial}{\partial z_{\epsilon}}, \\
& K\left(\frac{\partial}{\partial z_{\alpha}}, \frac{\partial}{\partial \bar{z}_{\beta}}\right)\left(\frac{\partial}{\partial \bar{z}_{\gamma}}\right)=\sum K_{\bar{\gamma} \alpha \bar{\beta}}^{\bar{\epsilon}} \frac{\partial}{\partial \bar{z}_{\epsilon}} .
\end{aligned}
$$

We have

$$
K_{\gamma a \bar{\beta}}^{\epsilon}=-\partial \Gamma_{a \gamma}^{\epsilon} / \partial \bar{z}_{\beta}, \quad K_{\bar{\gamma} \alpha \bar{\beta}}^{\bar{\epsilon}}=\partial \bar{\Gamma}_{\beta \gamma}^{\epsilon} / \partial z_{\alpha}
$$

The following formulas hold for covariant differentiation of $(p, q)$ forms:

$$
\begin{aligned}
& \left(\nabla_{\partial / \partial z_{\alpha}} \phi\right)_{A \bar{B}}=\frac{\partial}{\partial z_{\alpha}}\left(\phi_{A \bar{B}}\right)-\sum \Gamma_{a a_{s}}^{a} \phi_{a_{1} \cdots(a)_{s} \cdots a_{p} \bar{B}} \\
& \left(\nabla_{\partial / \partial \bar{z}_{\alpha}} \phi\right)_{A \bar{B}}=\frac{\partial}{\partial \bar{z}_{\alpha}}\left(\phi_{A \bar{B}}\right)-\sum \bar{\Gamma}_{a b_{t}}^{b} \phi_{A \bar{b}_{1} \cdots(\bar{b})_{t} \cdots \bar{b}_{q}}
\end{aligned}
$$

There is a naturally defined operator

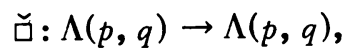

which we will call the reduced complex Laplacian. First define

$$
\check{\partial}: \Lambda(p, q) \rightarrow \Lambda(p, q+1)
$$

to be the alternization of covariant differentiation:

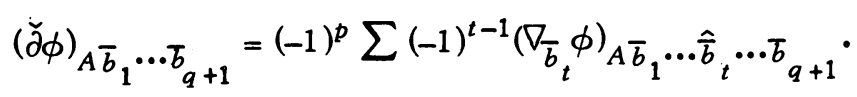

Now we normalize the metric $g_{\alpha \beta}=\delta_{\alpha \beta}$ at the origin of our coordinate system. The negative adjoint of $\breve{\partial}$ is given by

$$
* \# \check{\partial} \# * \text { = } \check{\theta} \text {, }
$$

where $*$ is the Hodge star operator, and \# is complex conjugation.

$$
(\check{\theta} \phi)_{A \bar{b}_{1} \cdots \bar{b}_{q-1}}=(-1)^{p} \sum\left(\nabla_{b} \phi\right)_{A \bar{b} \bar{b}_{1} \cdots \bar{b}_{q-1}} \cdot
$$


We define

$$
\check{u}=\check{\theta} \check{\partial}+\check{\partial} \check{\theta}
$$

$\check{\square}$ is a negative selfadjoint differential operator of order two. It is necessary to derive a simple formula for $\check{\text {. }}$. Now

$$
\begin{aligned}
& \left.(\check{\theta} \check{\partial} \phi)_{A \bar{B}}=\sum\left(\nabla_{b} \nabla_{\bar{b}} \phi\right)_{A \bar{B}}+\sum(-1)^{t} \nabla_{b} \nabla_{\bar{b}} \phi\right)_{t} \bar{b}_{\bar{b}} \ldots \hat{b}_{t} \ldots \bar{b}_{q}, \\
& (\check{\partial} \check{\theta} \phi)_{A \bar{B}}=\sum(-1)^{t-1}\left(\nabla_{\bar{b}} \nabla_{t} \phi\right)_{A \bar{b} \bar{b}} \ldots \hat{\bar{b}}_{t} \cdots \bar{b}_{q} .
\end{aligned}
$$

Thus

$$
(\check{\square} \phi)_{A \bar{B}}=\sum\left(\nabla_{b} \nabla_{\bar{b}} \phi\right)_{A \bar{B}}+\left(R_{p, q}(\phi)\right)_{A \bar{B}} \text {. }
$$

Here

$$
\begin{aligned}
& \left(R_{p, q}(\phi)\right)_{A \bar{B}}=\sum(-1)^{t-1}\left[\left(\nabla_{\bar{b}_{t}} \nabla_{b}-\nabla_{b} \nabla_{\bar{b}}\right) \phi\right]_{A} \bar{b}_{\bar{b}} \ldots \hat{\bar{b}}_{t} \ldots \bar{b}_{q} \\
& =\sum K_{a_{s} b b_{t}}^{a} \phi_{a_{1} \cdots(a)_{s} \cdots a_{p} b_{1} \cdots(b)_{t} \cdots b_{q}} \\
& +\sum K_{\bar{b}_{b} \bar{b}_{t}}^{\phi_{A \bar{b}} \cdots(\bar{\gamma})_{t} \cdots \bar{b}_{q}}+\sum K_{\bar{b}_{s} b \bar{b}_{t}^{\bar{\tau}}}^{\phi_{A b_{1}} \cdots(\bar{b})_{t} \cdots(\bar{\epsilon})_{s} \cdots \bar{b}_{q}} \cdot
\end{aligned}
$$

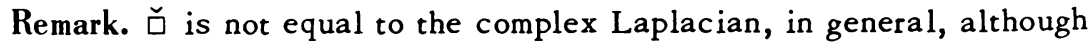
they coincide in the Kaehler case. More details may be found in [6].

2. The fundamental solution of the heat equation. $\breve{a}$ is a negative selfadjoint operator and is well known to have eigenvalues $0 \geq \lambda_{0}^{p, q} \geq \lambda_{1}^{p, q} \geq \cdots$, where each eigenvalue is repeated up to multiplicity. If $\phi_{i}^{p, q}$ are the corresponding eigenforms, then

$$
\sum \exp \left(t \lambda_{i}^{p, q}\right) \phi_{i}^{p, q}\left(z^{\prime}\right) \otimes \phi_{i}^{p, q}(z)
$$

converges uniformly on compact sets in $(0, \infty) \times M \times M$ to the fundamental solution of the heat equation:

$$
\left(\partial / \partial t-\check{\check{c}}_{z}\right) \phi=0 .
$$

In [4] Patodi constructs a parametrix for the fundamental solution of (2.2) on Kaehler manifolds. This derivation may be generalized to the Hermitian case without difficulty.

We first observe from the formula (1.2) that on functions $\check{0}$ has the same second order part as the real Laplacian. Also by (1.2) for any smooth function $f:$ 


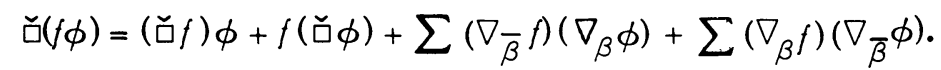

These are the basic facts which permit the derivation to go through unaltered. A sketch is given below.

Set

$$
H_{N}^{p, q}\left(t, z^{\prime}, z\right)=(2 \pi t)^{-d} \exp \left(\frac{-r^{2}}{2 t}\right) \sum_{i=0}^{N} t^{i} U^{i, p, q}\left(z^{\prime}, z\right),
$$

where $r$ is the geodesic distance between $z^{\prime}$ and $z, N>d$, and $U^{i, p, q}\left(z^{\prime}, z^{\prime}\right)$ are double $(p, q)$ forms defined in a normal neighborhood of the diagonal $\left(z^{\prime}, z^{\prime}\right)$.

Require the $U^{i, p, q}$ to satisfy the differential equation

$$
\left(\partial / \partial t-\check{\square}_{z}\right) H_{N}^{p, q}\left(t, z^{\prime}, z\right)=-(2 \pi t)^{-d} \exp \left(-r^{2} / 2 t\right) t^{N} \check{\check{L}}_{z} U^{N, p, q}\left(z^{\prime}, z\right)
$$

with the initial condition that $U^{0, p, q}\left(z^{\prime}, z^{\prime}\right)$ is the identity automorphism. This equation is equivalent to the system

$$
\nabla_{r / d r} U^{i, p, q}\left(z^{\prime}, z\right)+\left(i-d+1 / 2 \check{\square}_{z} r^{2}\right) U^{i, p, q}\left(z^{\prime}, z\right)=\check{\square}_{z} U^{i-1, p, q}\left(z^{\prime}, z\right),
$$

where $\nabla$ is covariant differentiation with respect to the Hermitian connection。

To solve the system of equations (2.4), we first observe that

$$
\left(1 / 2 \text { ă } r^{2}-d\right)\left(z^{\prime}, z^{\prime}\right)=\left(1 / 4 \Delta r^{2}-d\right)\left(z^{\prime}, z^{\prime}\right)=0,
$$

where $\Delta$ is the real Laplacian. Then $\left(1 / 2 \breve{a} r^{2}-d\right) / r$ approaches a finite limit as $r \rightarrow 0$ and is thus well defined.

Now rewrite the system (2.4) as follows:

$$
\nabla_{r}{ }_{r / d r}\left[U^{i, p, q}\left(z^{\prime}, z\right) r^{i} \exp \left(\int \frac{\left(1 / 2 \check{\square} s^{2}-d\right)}{s}\right)\right]=r^{i} \exp \left(\int \frac{\left(1 / 2 \check{\square} s^{2}-d\right)}{s}\right) \check{\square}_{z} U^{i-1, p, q}\left(z^{\prime}, z\right)
$$

with integration along the geodesic joining $z^{\prime}$ to $z$. Denote by $T_{z, w}$ the parallel translation with respect to the Hermitian connection. We have the following solution in a normal neighborhood of the diagonal:

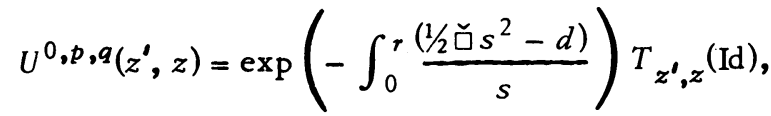

$$
\begin{aligned}
& U_{(2.5)}^{i, p, q}\left(z^{\prime}, z\right)=r^{-i} \exp \left[-\int_{0}^{r} \frac{\left(1 / 2 \check{L} s^{2}-d\right)}{s}\right] \\
& \cdot\left(\int_{0}^{r} s^{i-1} \exp \left[\int_{0}^{s} \frac{\left(1 / 2 \square t^{2}-d\right)}{t}\right] T_{U(s), z} \square_{z} U^{i-1, p, q}\left(z^{\prime}, z\right)\right),
\end{aligned}
$$


where $u(s)$ is the unique geodesic joining $z^{\prime}$ to $z$ lying in a normal neighborhood of the diagonal.

It is easy to see that the equations (2.5) determine smooth double forms in a sufficiently small neighborhood of the diagonal. Furthermore we may show that $H_{N}^{p, q}\left(t, z^{\prime}, z\right)$ contains the singular part of the fundamental solution for the heat equation (2.2). In particular

$$
\sum \exp \left(\lambda_{n}^{p, q} t\right)\left\langle\phi_{n}^{p, q}\left(z^{\prime}\right), \phi_{n}^{p, q}\left(z^{\prime}\right)\right\rangle=(2 \pi t)^{-d} \sum t^{i} \operatorname{Tr} U^{i, p, q}\left(z^{\prime}, z^{\prime}\right)+O(t) .
$$

We set $a_{i, p, q}\left(z^{\prime}\right)=\operatorname{Tr} U^{i, p, q}\left(z^{\prime}, z^{\prime}\right)$. The $a_{1, p, q}\left(z^{\prime}\right)$ will be computed in this paper.

3. Invariants on Hermitian manifolds. A bas is for the invariants of order two on Hermitian manifolds has been determined by Gilkey [3].

Theorem 3.1 (Gilkey). Let $M^{d}$ be a Hermitian manifold of complex dimension d. Define invariants of order two by

$$
\begin{aligned}
& W=\text { the scalar curvature, } \\
& W_{1}=*\left(d \bar{\partial} \Omega \wedge \Omega^{d-2}\right) \text { for } d \geq 2, \\
& W_{2}=b(\bar{\partial} \Omega, \partial \Omega) \text { for } d \geq 2, \\
& W_{3}=*\left(d \Omega \wedge \bar{\partial} \Omega \wedge \Omega^{d-3}\right) \text { for } d \geq 3 .
\end{aligned}
$$

Here $\Omega$ is the Kaehler form of the Hermitian metric $h$, and the other operators are as usual. Then

(a) If $d \geq 3, W, W_{1}, W_{2}, W_{3}$ are a basis for the invariants of order two.

(b) If $d=2, W, W_{1}, W_{2}$ are a basis for the invariants of order two and $W_{3}=0$.

(c) If $d=1, W$ is a basis for the invariants of order two and $W_{1}=W_{2}=$ $W_{3}=0$.

4. The coefficient $a_{1,0,0^{\circ}}$ By the equations (2.5) we have

$$
a_{1,0,0}=\check{\check{L}}_{z}\left(\exp -\int_{0}^{r}\left(\frac{\left(1 / 2 \breve{\square} s^{2}-d\right)}{s}\right) d s\right) \text {. }
$$

Now the real Laplacian is given by [5]:

$$
\Delta / 2=\check{u}+V, \quad V=-\frac{1}{2} \sum g^{i j} \Gamma_{i j}^{k} \frac{\partial}{\partial x_{k}} .
$$

Here $g^{i j}, \Gamma_{i j}^{k}(1 \leq i, j, k \leq 2 d)$ denote the usual quantities with respect to the Riemannian metric $g$ of $\oint 1$.

Then 


$$
\begin{aligned}
a_{1,0,0} & =\left(\frac{\Delta}{2}-V\right) \exp \left[\int_{0}^{r}\left(\frac{d-\Delta s^{2} / 4}{s}\right) d s+\int_{0}^{r}\left(\frac{V s^{2}}{2 s}\right) d s\right] \\
& =A+B+C+D+E+F
\end{aligned}
$$

where

$$
\begin{aligned}
& A=\frac{\Delta}{2} \exp \left(\int_{0}^{r} \frac{d-\Delta s^{2} / 4}{s} d s\right)=\frac{W}{6} \\
& B=\frac{\Delta}{2} \exp \left(\int_{0}^{r} \frac{V s^{2}}{2 s} d s\right)=W_{1}+\frac{9}{4} W_{2}+\frac{5}{2} W_{3}, \\
& C=\frac{\partial}{\partial z_{\alpha}} \exp \left(\int_{0}^{r} \frac{d-\Delta s^{2} / 4}{s} d s\right) \frac{\partial}{\partial \bar{z}_{\alpha}} \exp \left(\int_{0}^{r} \frac{V s^{2}}{2 s} d s\right)=0 \\
& D=\frac{\partial}{\partial \bar{z}_{\alpha}} \exp \left(\int_{0}^{r} \frac{d-\Delta s^{2} / 4}{s} d s\right) \frac{\partial}{\partial z_{\alpha}} \exp \left(\int_{0}^{r} \frac{V s^{2}}{2 s} d s\right)=0 \\
& E=-V \exp \left(\int_{0}^{r} \frac{d-\Delta s^{2} / 4}{s} d s\right)=0 \\
& F=-V \exp \left(\int_{0}^{r} \frac{+V s^{2}}{2 s} d s\right)=-1 / 2 W_{2}-W_{3} .
\end{aligned}
$$

The formula $A=W / 6$ is well known, since $A$ is just the term corresponding to $a_{1,0,0}$ in the asymptotic expansion for $\Delta / 2$. The expressions for $B, C, D, E, F$ follow by explicit computation. This computation requires only the expression

$$
r^{2}=\sum g_{i j} x_{i} x_{j}+\frac{1}{3} \sum\left(g_{l i} \Gamma_{j k}^{l}+g_{l j} \Gamma_{i k}^{l}+g_{l k} \Gamma_{j i}^{l}\right) x_{i} x_{j} x_{k}+\sum O\left(x_{i} x_{j} x_{k} x_{l}\right),
$$

which we derive in any real local coordinate system.

We have finally:

$$
a_{1,0,0}=W / 6+W_{1}+W_{2} / 4+3 W_{3} / 2 .
$$

5. The coefficient $a_{1, p, q^{*}}$ By the equations (2.5) we have for any $v \in \Lambda(p, q)$ :

$$
\begin{aligned}
U^{1, p, q}(0,0) v & =\check{\square}_{z}\left(\exp -\int_{0}^{r}\left(\frac{\left(1 / 2 \text { 口̆ } s^{2}-d\right)}{s}\right) T_{0, z}(v)\right) \\
& =\left(a_{1,0,0}\right) v+\sum \nabla_{b} \nabla_{\bar{b}} T_{0, z}(v)+R_{p, q}(v)
\end{aligned}
$$

by (1.2), (2.3). 
Now calculation using equations (1.1) yields:

$$
\begin{aligned}
& \left(\sum \nabla_{b} \nabla_{\bar{b}} T_{0, z}(v)\right)_{A \bar{B}}=-\frac{1}{2} \sum K_{a_{s} b b^{a}} V_{a_{1} \ldots(a)_{s} \cdots a_{p} \bar{B}}
\end{aligned}
$$

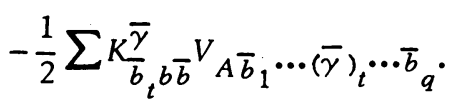

Thus

$$
\operatorname{Tr}\left(\sum \nabla_{b} \nabla_{\bar{b}} T\right)=-\frac{1}{2}\left(\begin{array}{l}
d-1 \\
p-1
\end{array}\right)\left(\begin{array}{l}
d \\
q
\end{array}\right) \sum K_{a b \bar{b}}^{a}-\frac{1}{2}\left(\begin{array}{l}
d \\
p
\end{array}\right)\left(\begin{array}{l}
d-1 \\
q-1
\end{array}\right) \sum K_{\bar{\gamma} b \bar{b}}^{\bar{\gamma}} .
$$

Also from the formulas (1.3) we obtain:

$$
\begin{aligned}
\operatorname{Tr}\left(R_{p, q}\right)= & \left(\begin{array}{l}
d-1 \\
p-1
\end{array}\right)\left(\begin{array}{l}
d-1 \\
q-1
\end{array}\right) \sum K_{a b \bar{b}}^{a}+\left(\begin{array}{l}
d \\
p
\end{array}\right)\left(\begin{array}{l}
d-1 \\
q-1
\end{array}\right) \sum K_{\bar{b} b \bar{\gamma}} \\
& +\left(\begin{array}{l}
d \\
p
\end{array}\right)\left(\begin{array}{l}
d-2 \\
q-2
\end{array}\right) \sum_{b \neq \epsilon} K_{\frac{\epsilon}{\epsilon} \bar{b}} .
\end{aligned}
$$

Expansion in terms of the derivatives of the metric gives:

$$
\begin{array}{ll}
\sum K_{a b \bar{b}}^{a}=W+W_{1}, & \sum K_{\bar{\gamma} b \bar{b}}^{\bar{\gamma}}=-W_{1}, \\
\sum K_{\bar{b}_{b \bar{\gamma}}}^{\bar{\gamma}}=-W-2 W_{1}+W_{2}, & \sum_{b \neq \epsilon} K_{\bar{\epsilon} b \bar{b}}^{\bar{\epsilon}}=W_{1}-W_{2} .
\end{array}
$$

Thus

$$
\begin{aligned}
a_{1, p, q}= & a_{1,0,0}\left(\begin{array}{l}
d \\
p
\end{array}\right)\left(\begin{array}{l}
d \\
q
\end{array}\right)+W\left(-\frac{1}{2}\left(\begin{array}{l}
d-1 \\
p-1
\end{array}\right)\left(\begin{array}{l}
d \\
q
\end{array}\right)-\frac{1}{2}\left(\begin{array}{l}
d \\
p
\end{array}\right)\left(\begin{array}{l}
d-1 \\
q-1
\end{array}\right)+\left(\begin{array}{l}
d-1 \\
p-1
\end{array}\right)\left(\begin{array}{l}
d-1 \\
q-1
\end{array}\right)\right) \\
& +W_{1}\left(-\frac{1}{2}\left(\begin{array}{l}
d-1 \\
p-1
\end{array}\right)\left(\begin{array}{l}
d \\
q
\end{array}\right)-\frac{3}{2}\left(\begin{array}{l}
d \\
p
\end{array}\right)\left(\begin{array}{l}
d-1 \\
q-1
\end{array}\right)+\left(\begin{array}{l}
d \\
p
\end{array}\right)\left(\begin{array}{l}
d-2 \\
q-2
\end{array}\right)+\left(\begin{array}{l}
d-1 \\
p-1
\end{array}\right)\left(\begin{array}{l}
d-1 \\
q-1
\end{array}\right)\right) \\
& +W_{2}\left(\left(\begin{array}{l}
d \\
p
\end{array}\right)\left(\begin{array}{l}
d-1 \\
q-1
\end{array}\right)-\left(\begin{array}{l}
d \\
p
\end{array}\right)\left(\begin{array}{l}
d-2 \\
q-2
\end{array}\right)\right) \\
= & W\left(\frac{1}{6}\left(\begin{array}{l}
d \\
p
\end{array}\right)\left(\begin{array}{l}
d \\
q
\end{array}\right)-\frac{1}{2}\left(\begin{array}{l}
d-1 \\
p-1
\end{array}\right)\left(\begin{array}{l}
d \\
q
\end{array}\right)-\frac{1}{2}\left(\begin{array}{l}
d \\
p
\end{array}\right)\left(\begin{array}{l}
d-1 \\
q-1
\end{array}\right)+\left(\begin{array}{l}
d-1 \\
p-1
\end{array}\right)\left(\begin{array}{l}
d-1 \\
q-1
\end{array}\right)\right) \\
& +W_{1}\left(\left(\begin{array}{l}
d \\
p
\end{array}\right)\left(\begin{array}{l}
d \\
q
\end{array}\right)-\frac{1}{2}\left(\begin{array}{l}
d-1 \\
p-1
\end{array}\right)\left(\begin{array}{l}
d \\
q
\end{array}\right)+\left(\begin{array}{l}
d \\
p
\end{array}\right)\left(\begin{array}{l}
d-2 \\
q-2
\end{array}\right)\right. \\
& +W_{2}\left(\frac{7}{4}\left(\begin{array}{l}
d \\
p
\end{array}\right)\left(\begin{array}{l}
d \\
q
\end{array}\right)+\left(\begin{array}{l}
d \\
p
\end{array}\right)\left(\begin{array}{l}
d-1 \\
q-1
\end{array}\right)-\left(\begin{array}{l}
d \\
p
\end{array}\right)\left(\begin{array}{l}
d-2 \\
q-2
\end{array}\right)\right)+W_{3}\left(\frac{3}{2}\left(\begin{array}{l}
d \\
p
\end{array}\right)\left(\begin{array}{l}
d \\
q
\end{array}\right)\right) .
\end{aligned}
$$


Remark. In the Kaehler case this agrees with the computations of the author [2] and Gilkey [3]. Different methods were used in each paper.

Remark. The coefficients $a_{i, p, q}$ satisfy Serre duality, $a_{i, p, q}=$ $a_{i, d-p, d-q}$, as a consequence of the definition of the reduced complex Laplacian. This is verified by calculation in the above special case.

Theorem 5.1. (a) Suppose $M$ and $M^{\prime}$ are complex manifolds isospectral on $(p, q)$ forms with respect to the reduced complex Laplacian. Then $M$ is Kaehler if and only if $M^{\prime}$ is Kaehler.

(b) Suppose $M$ is a complex manifold such that the reduced complex Laplacian and the real Laplacian divided by two have the same spectrum on forms. Then $M$ is Kaehler.

Proof. (a) $\int a_{1, p, q}=\int a_{1, p, q^{-}}^{\prime}$ The equation $\int W_{2}^{\prime}=\int W_{2}=0$ is an algebraic consequence of these equations with $(p, q)=(0,0),(0,1),(1,0)$, and the relation $\int W_{1}=-(d-2) \int W_{3}$ given by Gilkey [3].

(b) The proof is similar. We need only consider the coefficients on functions and one forms.

\section{REFERENCES}

1. Marcel Berger, Eigenvalues of the Laplacian, Proc. Sympos. Pure Math., vol. 16, Amer. Math. Soc., Providence, R. I., 1970, pp. 121-125. MR 41 \#9141.

2. Harold Donnelly, Minakshisundaram's coefficients on Kaehler manifolds, Proc. Sympos. Pure Math., vol. 27, Amer. Math. Soc., Providence, R. I. (to appear).

3. Peter Gilkey, Spectral geometry and the Kaehler condition for complex manifolds (to appear).

4. V. K. Patodi, An analytic proof of the Riemann-Roch-Hirzebruch theorem for Kaehler manifolds, J. Differential Geometry 5 (1971), 251-283. MR 44 \#7502.

5. - Curvature and the eigenforms of the Laplace operator, J. Differential Geometry 5 (1971), 233-249. MR 45 \#1201.

6. E. Vesentini, Lectures on Levi convexity and cohomology vanishing theorems, Tata Inst. of Fund. Res. Lect. on Math., no. 39, Tata Institute of Fundamental Research, Bombay, 1967. MR 38 \#342.

DEPARTMENT OF MATHEMATICS, UNIVERSITY OF CALIFORNIA, BERKELEY, CALIFORNIA 94720

Current address: Department of Mathematics, Massachusetts Institute of Technology, Cambridge, Massachusetts 02139 